\title{
Axon Pathway Boundaries in the Developing Brain. I. Cellular and Molecular Determinants That Separate the Optic and Olfactory Projections
}

\author{
Jerry Silver, Michael Poston, and Urs Rutishauser \\ Neuroscience Program, Department of Developmental Genetics, Case Western Reserve University School of Medicine, \\ Cleveland, Ohio 44106
}

\begin{abstract}
When optic fibers first approach the chiasmatic region of the diencephalon in the chick embryo on days 3 and 4 (E34), they rarely grow rostrally into the olfactory region of the telencephalon. Conversely, olfactory tract axons grow as far as, but never cross the diencephalic/telencephalic (D/T) boundary to enter the optic chiasm. In this study, a region of specialized neuroepithelium, originally named the "knot" in mouse by Silver (1984), has been identified at the $D / T$ border of chick embryos. At pre-axonal stages, the presumptive knot region undergoes a cataclysmic cell death, with concomitant phagocytosis of necrotic debris by the remaining cells. When fibers subsequently appear in the chiasm and olfactory tracts, the knot consists of a very dense, interwoven cluster of non-neuronal cells that lack marginal radial processes, and whose cell bodies directly abut the glial limiting membrane. Thus, the morphology of the knot is in sharp contrast to the cell body-free marginal zone and endfoot regions along which axons tend to grow. In addition, we found that the neural cell adhesion molecule (N-CAM), which is expressed on neuroepithelial cell processes within the central optic and olfactory pathways, is not present on cells in the knot region during periods of axon growth. These results suggest that the knot, through its elimination of the marginal zone processes, absence of large extracellular spaces, and relative absence of adhesion molecules, functions as an axon-refractory barrier that effectively separates the optic and olfactory projections.
\end{abstract}

When the first retinal ganglion cell axons arrive at the floor of the developing optic chiasm in the primitive diencephalon, there are at least 4 potential avenues of growth available to them (Fig. 1). Each of these possible choices diverges from the chiasm along discrete segments of one continuous sheet of neuroepithelial tissue. Two of the pathways, one on each side of the midline, lead directly into the optic tracts, to the thalami and tecta; a third passes into the optic nerve of the opposite eye; and a fourth merges directly with the olfactory region of the basal telencephalon. Optic axons grow preferentially at the neural margin (Silver

Received Sept. 17, 1986; revised Dec. 30, 1986; accepted Dec. 30, 1986.

This research was supported by Grants EY-05952, EY-06107, and HD-18369 from the NIH and Grant GC-A-607555 from the National Science Foundation. The authors wish to express their gratitude to Catherine Doller for her technical assistance.

Correspondence should be addressed to Jerry Silver at the above address.

Copyright (C) 1987 Society for Neuroscience $0270-6474 / 87 / 072264-09 \$ 02.00 / 0$ and Sapiro, 1981; Silver and Rutishauser, 1984), but do so primarily along the first 2 of these corridors (Herrick, 1941; Stone, 1960; Goldberg, 1974; Lund and Bunt, 1976; Schwenk and Hibbard, 1977; Rager and von Oeynhausen, 1979; Steedman et al., 1979; Ehrlich and Mark, 1984; Holt, 1984) and transiently along the third (McLoon and Lund, 1982; O'Leary et al., 1983). However, in birds and mammals optic axons seldom, if ever, extend rostrally into the telencephalon to enter the nearby olfactory pathway (Kallen, 1954; Pickard and Silverman, 1981). Conversely, olfactory tract axons, which also grow at the margin, rarely breach the boundary between the caudal telencephalon and the closely juxtaposed rostral border of the chiasm (Kallen, 1954). This basic segregation of the developing olfactory and optic projections within the marginal zone becomes a prominent and permanent feature of the brain in warm-blooded vertebrates. As a result, the olfactory system lacks direct connections with the thalamus, and the optic system does not establish a primary innervation with the frontal cortex (Brodal, 1981).

Although axon tracts usually occupy stereotyped positions, a number of studies have shown that transplanted retinal ganglion cells will generate axons that grow into regions of the CNS that are far beyond their usual borders, such as the spinal cord (Constantine-Paton and Capranica, 1976; Katz and Lasek, 1979; Harris, 1980). Host optic axons in young rodents will also leave the normal optic pathway to invade transplanted pieces of embryonic colliculus when the graft is placed near the host tectum (Harvey and Lund, 1981; Alvarado-Mallart and Sotelo, 1984). Even within such ectopic regions, optic fibers still tend to grow preferentially at the outer marginal zone along reproducible routes. Since optic axons have the ability to grow in foreign regions of the brain (also see Frost, 1984), why, then, don't optic fibers spread incessantly beyond their usual borders, for instance, forward into olfactory territories, during normal development? Are there any morphological or molecular characteristics of the cells at the presumptive diencephalic/telencephalic $(\mathrm{D} / \mathrm{T})$ border that distinguish them from the cells that lie along the terrain where axons are found?

In an attempt to address these questions, we have analyzed the $\mathrm{D} / \mathrm{T}$ junction in developing chicks prior to, during, and after the arrival of the earliest optic nerve fibers and olfactory tract axons at this important, axon-free landmark. In previous studies of the embryonic mouse, a "knotlike" structure composed of primitive glial cells was discovered at this location (Silver, 1984; Silver et al., 1985). Here we describe in detail the cellular, as well as molecular and developmental, peculiarities of a similar 
structure in the chick brain and relate them to the behavior of axons at the $\mathrm{D} / \mathrm{T}$ junction.

\section{Materials and Methods}

White Leghorn chicken embryos were incubated in a humidified chamber at $38^{\circ} \mathrm{C}$ for $2-5 \mathrm{~d}$ and then staged according to Hamburger and Hamilton (1951). Those embryos at the specific stages between 12 (embryonic day 2, E2) and 27 (E5) were then stripped of overlying membranes and decapitated. The heads were immersion-fixed and processed for either light-microscopic, transmission electron-microscopic (TEM), or immunohistochemical observation.

Embryos that were to be embedded in paraffin were fixed for 2-3 hr at room temperature, and then overnight at $4^{\circ} \mathrm{C}$, in Bodian's fixative ( $90 \mathrm{ml} ; 80 \% \mathrm{EtOH}, 5 \mathrm{ml}$ glacial acetic acid, $5 \mathrm{ml}$ formalin). The tissue was dehydrated through absolute EtOH, placed in xylene, and then infiltrated with and embedded in paraffin. Blocks were sectioned sagittally at $10 \mu \mathrm{m}$ on a microtome and the sections placed on albumincoated slides, dried, stained with hematoxylin, and then viewed with a Zeiss light microscope.

Those embryos to be embedded in plastic for thin-sectioning were fixed for 3-4 hr at room temperature in a $0.1 \mathrm{M}$ sodium cacodylate buffer containing 3\% glutaraldehyde, $1 \%$ paraformaldehyde, $1 \%$ acrolein, plus $10 \mathrm{~mm}$ each of $\mathrm{CaCl}, \mathrm{MgCl}, \mathrm{KCl}, \mathrm{NaCl}$, and dextrose. Tissues were rinsed in buffer, postfixed in $1 \% \mathrm{OsO}_{4}$ in buffer at $4^{\circ} \mathrm{C}$ for $2 \mathrm{hr}$, rinsed again, and then dehydrated and embedded in Spurr's plastic. Serial $1-\mu \mathrm{m}$-thick sections of optic nerve and chiasm were taken with a Sorvall MT-2B microtome, stained with toluidine blue to visualize histological features, and viewed with a Zeiss light microscope. Ultrathin (70-90 A) sections of selected areas were taken with a DuPont MT5000 ultramicrotome, mounted on Formvar-coated mesh grids, stained with lead citrate and uranyl acetate, and viewed with a Zeiss 109 electron microscope.

Embryos to be frozen-sectioned and stained with antibodies were fixed for $2 \mathrm{hr}$ at room temperature in $4 \%$ paraformaldehyde in PBS. Tissues were then rinsed in buffer and permeated overnight at $4^{\circ} \mathrm{C}$ with a solution of $20 \%$ sucrose in buffer to cryoprotect the tissue. The tissues were then quickly frozen in OCT embedding medium (Tissue Tek, Miles Scientific) using dry ice and acetone, then serially sectioned at $10 \mu \mathrm{m}$ in the sagittal or coronal plane with a Hacker cryostat. The sections were collected on gelatin-coated slides and incubated with $20 \mathrm{~mm}$ lysine for $1 / 2 \mathrm{hr}$ at room temperature to help eliminate potential cross-linking of antibody by residual aldehydes from the fixative. After being rinsed in PBS, the sections were incubated in $10 \%$ normal goat serum for $1 / 2 \mathrm{hr}$ at room temperature to help reduce possible nonspecific background adsorption of the secondary antibody. Sections were rinsed again in PBS, and then incubated with a 1:200 dilution of monoclonal antibodies against neural cell adhesion molecule (N-CAM) in buffer plus $10 \%$ goat serum for 1 $\mathrm{hr}$ at room temperature. After the sections were rinsed, they were incubated for $1 \mathrm{hr}$ at room temperature with the secondary antibody, a peroxidase-conjugated, goat anti-mouse IgG, diluted 1:100 in PBS plus $10 \%$ goat serum. The monoclonal antibodies used (Mab-PP) are directed against an extracellular polypeptide epitope present on all forms of $\mathrm{N}$ CAM (Frelinger and Rutishauser, 1986; Watanabe et al., 1986). After rinsing away the secondary antibody with PBS, the sections were incubated at room temperature with $40 \mathrm{mg}$ of diaminobenzidine and 40 $\mu \mathrm{l}$ of $30 \% \mathrm{H}_{2} \mathrm{O}_{2}$ in $200 \mathrm{ml}$ of Tris base $(0.05 \mathrm{M}, \mathrm{pH} 7.5)$ until a brown reaction product appeared on the sections that had been exposed to the primary antibody. Slides containing the sections were thoroughly rinsed in PBS, coverslipped in Aquamount, and viewed with a Zeiss light microscope equipped with differential-interference contrast optics.

\section{Results}

In chick, the first wave of optic fibers from the retinal ganglion cells appears in the proximal stalk (not shown) and at the primitive chiasm late on embryonic day 3 or early on embryonic day 4 . At these early stages, the axons can be detected either by electron microscopy (Fig. $2 C$ ) or by staining with antibodies to N-CAM (Fig. 2D). Also, by the end of day 3 of gestation, ample numbers of fibers are present at the base of the diencephalon and in the tract, so that fascicles can be visualized by light microscopy of toluidine blue-stained plastic sections (Fig. $2 B$ ). At this stage the chiasmatic fibers are all located at the brain's
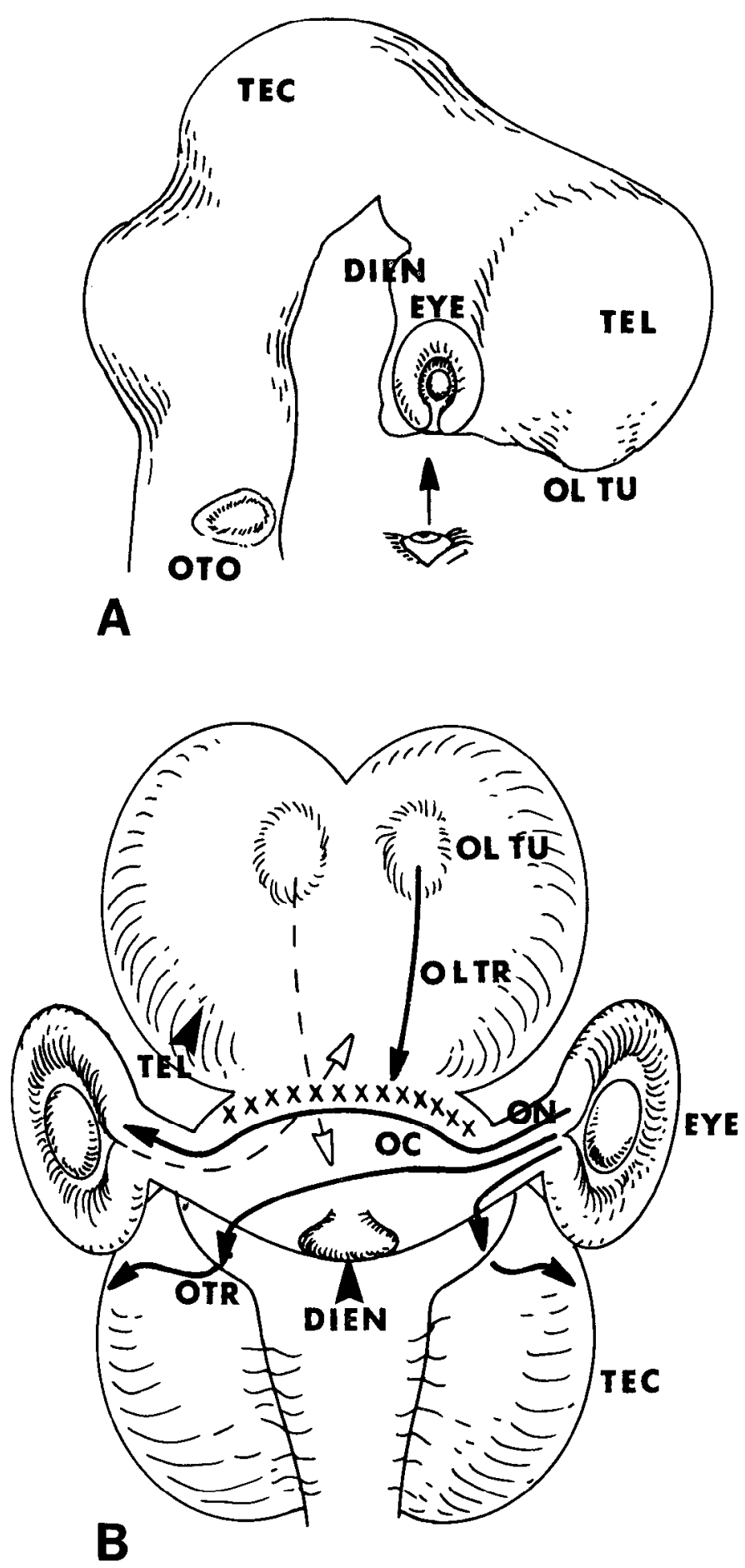

Figure 1. Sagittal and ventral surface views of an embryonic chick brain at $72 \mathrm{hr}$ of gestation showing some gross anatomical landmarks that can be used for orientation. $A$, Sagittal view showing the diencephalic/telencephalic junction (arrow), as well as the eye cup and olfactory tubercle $(B)$. Ventral view of a $72 \mathrm{hr}$ chick brain at the level of the viewer's eye shown under $A$. $B$, Some of the various routes known to be taken by the central fibers of the olfactory and optic systems during development shown by solid black arrows. Two additional potential routes for the olfactory and optic axons across the boundary between the diencephalon and telencephalon (row of $\times$ 's) are indicated by the dashed, open arrows. Note, however, that few, if any, fibers actually cross the $\mathrm{D} / \mathrm{T}$ junction. TEC, Tectum; DIEN, diencephalon; $T E L$, telencephalon; $O T O$, otocyst; $O L T U$, olfactory tubercle; $O L T R$, olfactory tract; $O N$, optic nerve; $O C$, optic chiasm; $O T R$, optic tract. 
Figure 2. A, Sagittal section through the $\mathrm{D} / \mathrm{T}$ junction region of an $\mathrm{E} 2.5$ chick stained with a monoclonal antibody to N-CAM and photographed with DIC optics. Note the patch of N-CAM staining (arrow) immediately caudal to the zone of necrosis $(N)$ (the future knot region), adjacent to the pre-optic recess $(P R) . B$, Sagittal plastic section $(1 \mu \mathrm{m})$ at the D/T junction of an E3.5 chick, showing the marginal row of axon fascicles at the chiasm. The axon fascicles are located caudal to some remaining pycnotic debris in cells at the $\mathrm{D} / \mathrm{T}$ junction. Note the progressive decrease in the width of the marginal zone and the amount of extracellular space moving from the tract (1) through the chiasm (2) and forward to the $\mathrm{D} / \mathrm{T}$ junction (3). $C$, Electron micrograph of a fascicle of axons at the chiasm, wrapped by glial cell processes. The small fingerlike extensions of glia (arrow) tend to lack organelles. $D$, Higher-power sagittal section of the chiasm early on E4 (low magnification in Fig. 4) showing the staining pattern for N-CAM by brightfield illumination. The marginal crescent of axon fascicles $(A X)$ at the chiasm is positive for N-CAM, as is the group of radial neuroepithelial cells $(N E)$ that makes contact with the axons. Immediately forward of the rostralmost fascicle is the pre-optic recess $(P R)$ and $\mathrm{D} / \mathrm{T}$ junction. This zone lacks N-CAM determinants (asterisk). E , Coronal section of the chick chiasm early on E4, stained with N-CAM antibodies, and observed by bright-field illumination. This figure demonstrates that the concentration of N-CAM on axons, as well as on support cells (arrow), at the chiasm appears to be highest at the endfoot margin. $A, \times 600 ; B, \times 300 ; C, \times 20,000$; $D, \times 300 ; E, \times 350$.
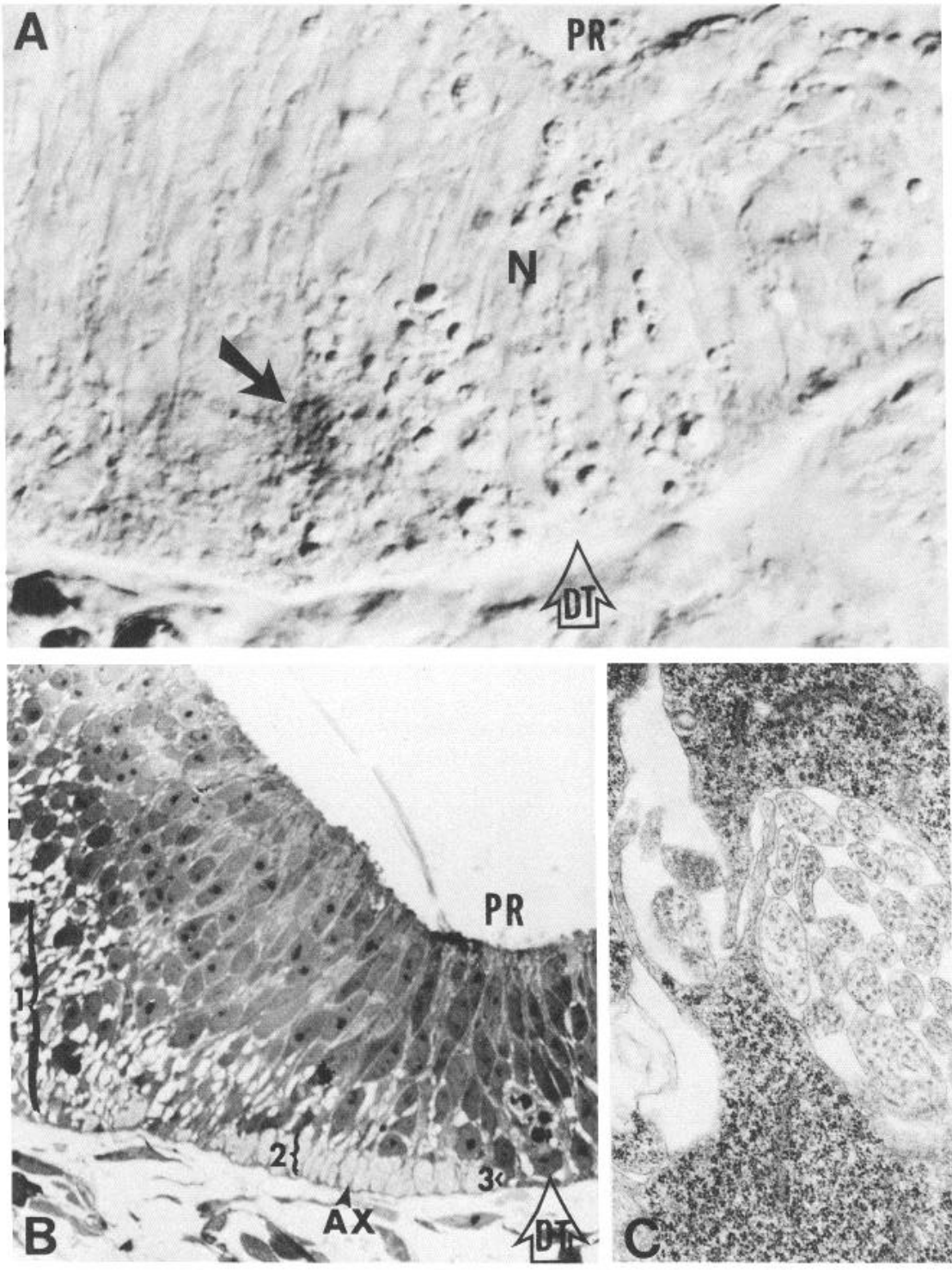

D
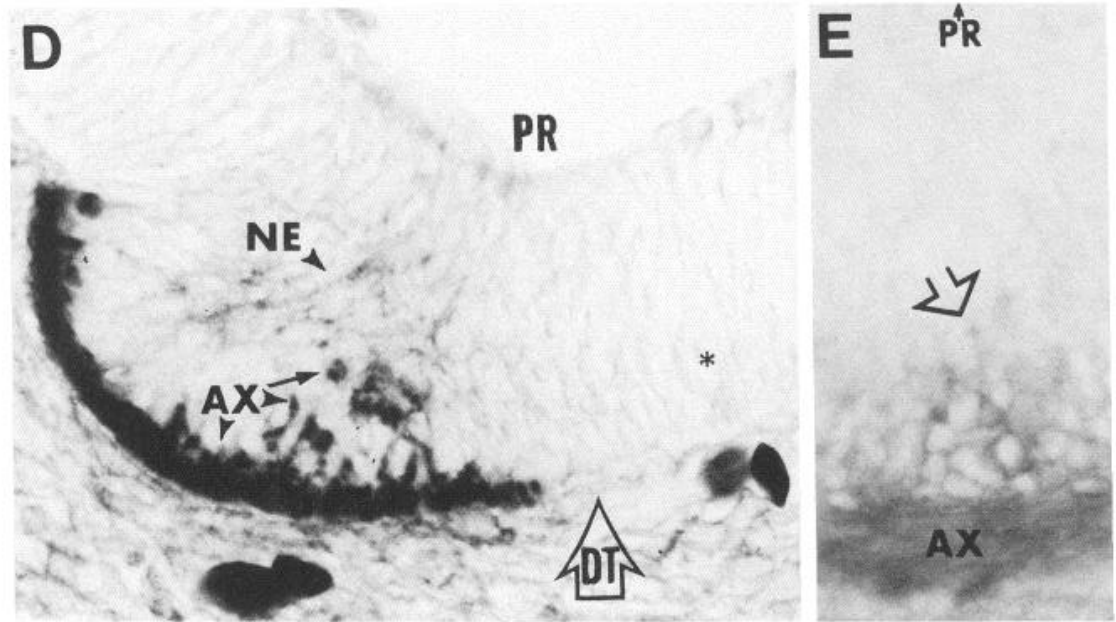

outer margin, bundled into a single, crescent-shaped row of approximately 2 dozen fascicles, each containing about 30-75 axons (Fig. 2B).

The gross positioning of the entire fascicle array at the chiasm is highly stereotyped, particularly at its front edge. In the sagittal plane, the rostralmost fascicle is always positioned precisely at the $\mathrm{D} / \mathrm{T}$ junction (Figs. $2 B, D ; 4 A ; 5 B$ ). This landmark is easily located because of a small $\mathrm{v}$-shaped kink, the pre-optic recess, that develops at the ventralmost border of the lamina terminalis, subjacent to the third ventricle (Fig. $3 A$ ). The recess is present 

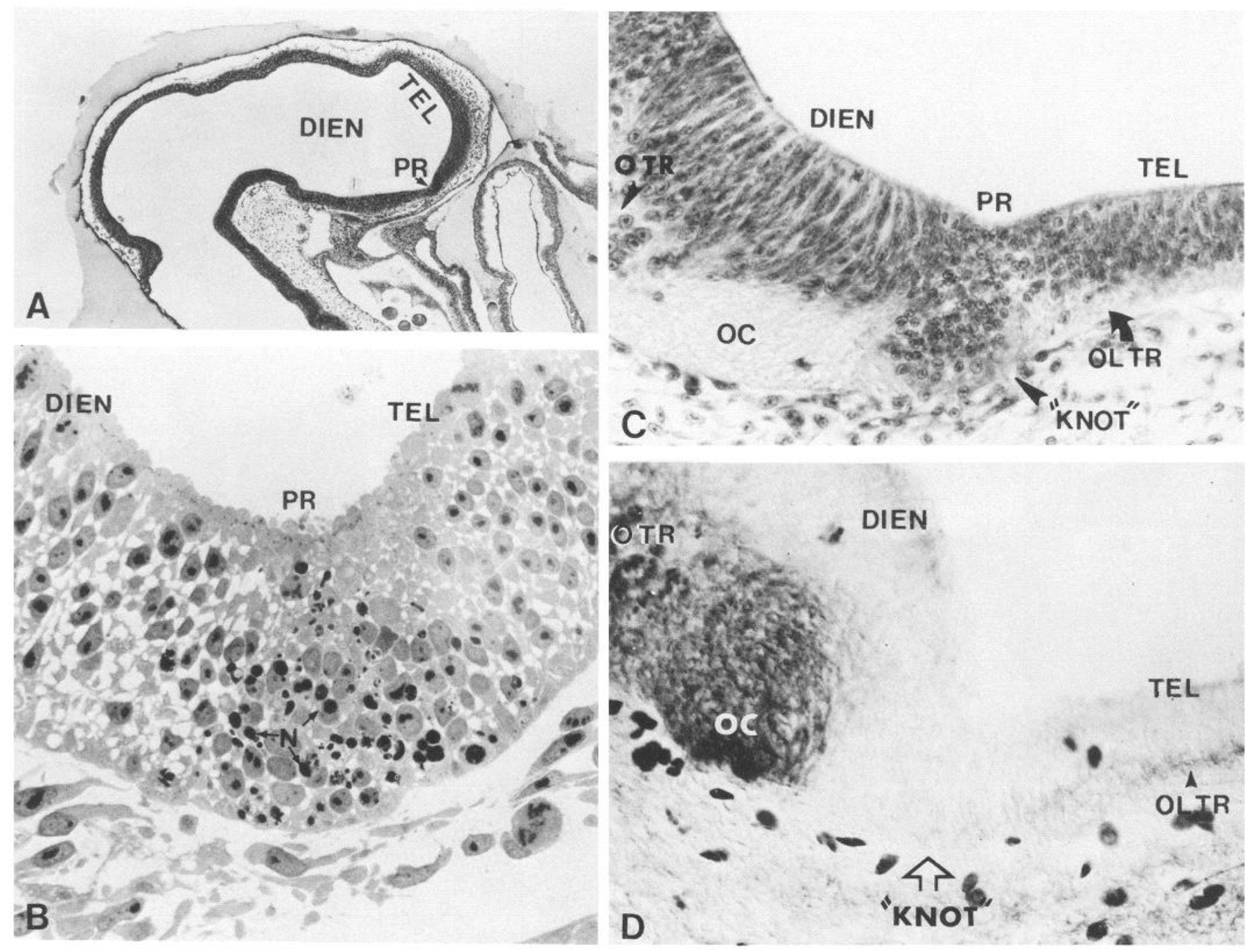

Figure 3. Midsagittal sections of the chiasmatic regions of E2 $(A, B)$ versus E5 $(C, D)$ chicks. When compared, sections demonstrate the changes that occur in the morphology and distribution of N-CAM at the D/T junction at a pre- and postaxonal stage. $A, \mathrm{~A} 1 \mu \mathrm{m}$ plastic section through an E2 chick brain, showing the pre-optic recess $(P R)$ that demarcates the telencephalic brain vesicle $(T E L)$ from the diencephalic vesicle $(D I E N)$. $B$, Higher-power micrograph of the pre-optic recess region (low magnification in $A$ ) showing the large amount of pycnosis (arrows) present in this region of the neuroepithelium during this stage. Note the greater abundance of extracellular spaces adjacent to, rather than within, the zone of cell death. $C$, Sagittal section through the $\mathrm{D} / \mathrm{T}$ junction and pre-optic recess $(P R)$ at $\mathrm{E} 5$, showing the dense knot of cells that lies between the rostral end of the optic chiasm $(O C)$ and the caudal end of the olfactory tract $(O L T R)$. D. A section comparable to that in $C$, demonstrating the absence of N-CAM staining in the knot region of an E5 chick (OTR, optic tract). $A, \times 25 ; B, \times 400 ; C, \times 250 ; D, \times 250$.

early on embryonic day 2 , well before optic fibers are generated, and thus serves as a pre-axonal landmark of the presumptive chiasm (Fig. 3A). By embryonic day 4 , fibers of the olfactory tract have also grown far enough caudally to be visualized near the D/T junction. They, like the optic fibers, come very close to, but do not pass through the pre-optic recess portion of the lamina terminalis (Fig. 4A).

Morphological differences between areas at the chiasm where axons do or do not grow. Serial section analyses of the tissue that forms the pre-optic recess, the border between the optic and olfactory projections, reveal that the neuroepithelium within this region is morphologically distinct from the axon-rich neuroepithelium on either side. On embryonic days 3 and 4 , the neuroepithelial cells within the boundaries of the optic and olfactory tracts develop relatively lengthy radial processes that are continuous with the pial endfeet (Figs. $2 B ; 5 A$ ). In addition, light-microscopic examination of the floor of the forebrain on embryonic days 3 and 4 reveals a decreasing gradation in the length of marginal neuroepithelial processes as one moves from the tract through the chiasm, and approaches the $\mathrm{D} / \mathrm{T}$ junction. This phenomenon is most easily demonstrated on E3.5, in parasagittal sections that pass through the optic tract (axons present), chiasm (axons present), and the pre-optic recess (axons absent). At this stage, the length of the radial processes changes progressively from about $60-75 \mu \mathrm{m}$ in the tract to approximately $5-10 \mu \mathrm{m}$ in the chiasm and $0-0.5 \mu \mathrm{m}$ at the $\mathrm{D} / \mathrm{T}$ junction (Fig. $2 B$ ). Also, at early stages and with routine fixation with mixed aldehydes, the radiating processes in the tract and chiasm, but not at the D/T border, appear to be separated from one another by wide (4-7 $\mu$ m diameter) extracellular spaces, which have been shown to be filled with an extracellular matrix (Krayanek, 1980).

On the following day (E5), cells at the $\mathrm{D} / \mathrm{T}$ junction begin to aggregate into a dense cluster that, because of its distinctive morphology, has been termed the "knot." An exceptional example of the knot, lying at the level of the pre-optic recess between the optic and olfactory projections, is shown in Figure 

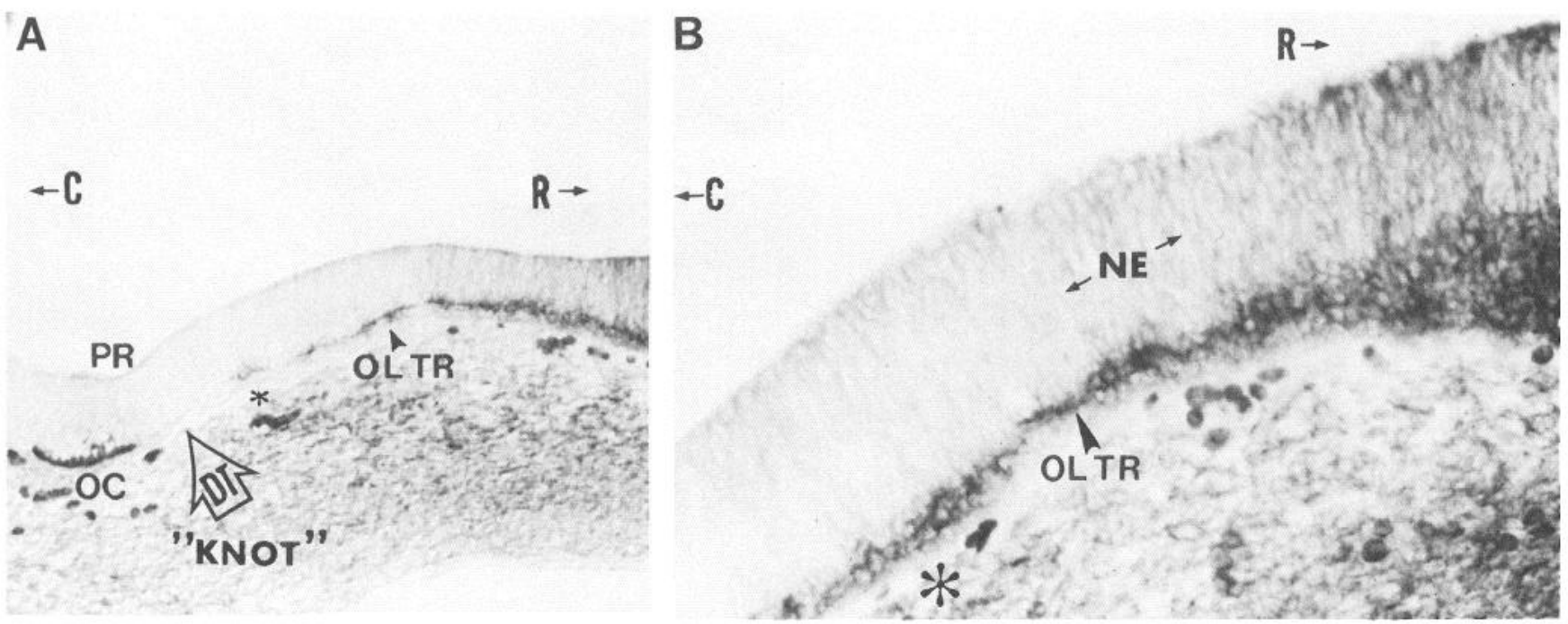

Figure 4. A, Sagittal section, at $40 \times$ magnification, of an E4 chick brain stained with antibodies to N-CAM, showing the overall distribution of N-CAM determinants at the optic chiasm $(O C)$ and olfactory tract $(O L T R)$ separated by the knot. $A, B(250 \times$ magnification), Note the progressive increase in intensity and radial spread of N-CAM staining on neuroepithelial cells $(N E)$ moving from caudal $(C$-arrow) to rostral $(R$-arrow) along the olfactory tract. The staining in the caudalmost portion of the olfactory tract (asterisks) is primarily at the pial endfoot margin. The staining in this region probably represents a combination of N-CAM determinants that are on the leading front of olfactory tract axons plus that on the marginal processes and endfeet of neuroepithelial cells.

$3 C$. In this particular embryo, the knot has developed to a large size; however, even in specimens where the knot is less developed, there is always a dramatic difference in cell organization within its structure relative to that of the chiasmic and tract regions.

Figure 5 illustrates the ultrastructural differences in supportcell morphology and axonal patterning in these various zones in a day 5 embryo. Cells in the region of the knot lack any recognizable radially oriented elements, and instead extend their processes into a dense, meshlike network. Also, in contrast to the chiasm and tract, the cell somata in the knot are closely packed and lie directly adjacent to the external limiting membrane. In all embryos at this stage, the most rostrally located axon fascicle at the chiasm is situated immediately caudal to the knot. In the 8 animals examined, no axon fascicles were found within or traveling through this structure to cross the $\mathrm{D} / \mathrm{T}$ junction (compare Fig. 5, $A$ and $B$ ). It remains possible, however, that our methods would not have detected a few axons or growth cone filopodia probing into or passing through the $\mathrm{D} / \mathrm{T}$ border.

Between embryonic days 3.5 and 5 , axon fascicles at the chiasm accumulate caudally and radially until they nearly fill the sector of tissue that lies between the knot and the primitive tract (Fig. $3 D$ ). Each fiber fascicle at the chiasm is tightly bundled by enwrapping glial processes and maintained in close association with neighboring fascicles (Fig. 2C). In contrast, optic axons in the tract spread out dramatically from their highly confined arrangement at the chiasm to occupy all regions of the elongated marginal zone (Fig. 5A). It is noteworthy that, although the marginal processes of neuroepithelial cells along the optic pathway reach their maximum length in the tract, they tend to maintain their primitive radial arrangement, have relatively fewer axon-enwrapping processes than they did in the chiasm, and remain separated by large (3-5 $\mu \mathrm{m}$ ) extracellular spaces even in the presence of axons. Striking differences in cytoskeletal content and rearrangements of axons, which may be related to the morphological differences described here, have also recently been reported within glial domains in the optic tract and chiasm of fish (Maggs and Scholes, 1986).

\section{Cell death within the presumptive knot region}

Do any peculiar events occur within the $\mathrm{D} / \mathrm{T}$ junction before the arrival of optic axons, and even prior to the onset of the more dramatic histogenetic changes that form the knot? In order to answer this question, we examined serial sagittal sections taken through the primitive $\mathrm{D} / \mathrm{T}$ junction early on embryonic day 2 , well before optic axons appear within the eye. The presumptive knot region is easily identifiable because of its colocalization with the pre-optic recess (Fig. $3 A$ ).

A striking feature at this early stage was the presence of an intense, focal necrosis within the neuroepithelium, precisely at the D/T junction (Figs. 3B, 6). At the ultrastructural level, a large amount of debris was clearly evident in pycnotic vacuoles throughout the cytoplasm of viable neuroepithelial cells (Fig. 6). Apparently, the bulk of the pycnosis is phagocytosed locally, since there was no evidence of macrophage invasion within the necrotic region. During the peak period of cell death (E2-3), and with our fixation protocol, the density of the tissue appeared greater (i.e., with less extracellular space) in the region undergoing necrosis than in adjacent areas that were not necrotic (Fig. $3 B)$.

\section{Distribution of $\mathrm{N}-\mathrm{CAM}$ within and adjacent to the primitive knot}

Figures $2, A, D$, and $3 D$ illustrate representative sagittal sections taken at equivalent levels through the chick optic chiasm, tract, and basal telencephalon at days $2.5,4$, and 5 of gestation. Also, in order to easily appreciate the changes that occur at the D/T junction at pre- and post-optic axonal stages, the morphology of the chiasm at E2 is compared with that on E5 in Figure 3. In the day 2 embryo, a pre-axonal stage for retinal ganglion cells, $\mathrm{N}$-CAM antigenic determinants at the primitive chiasm were detected only at or near the ventral, pial margin. In the example shown (Fig. $2 A$ ), the N-CAM is focused immediately caudal to 


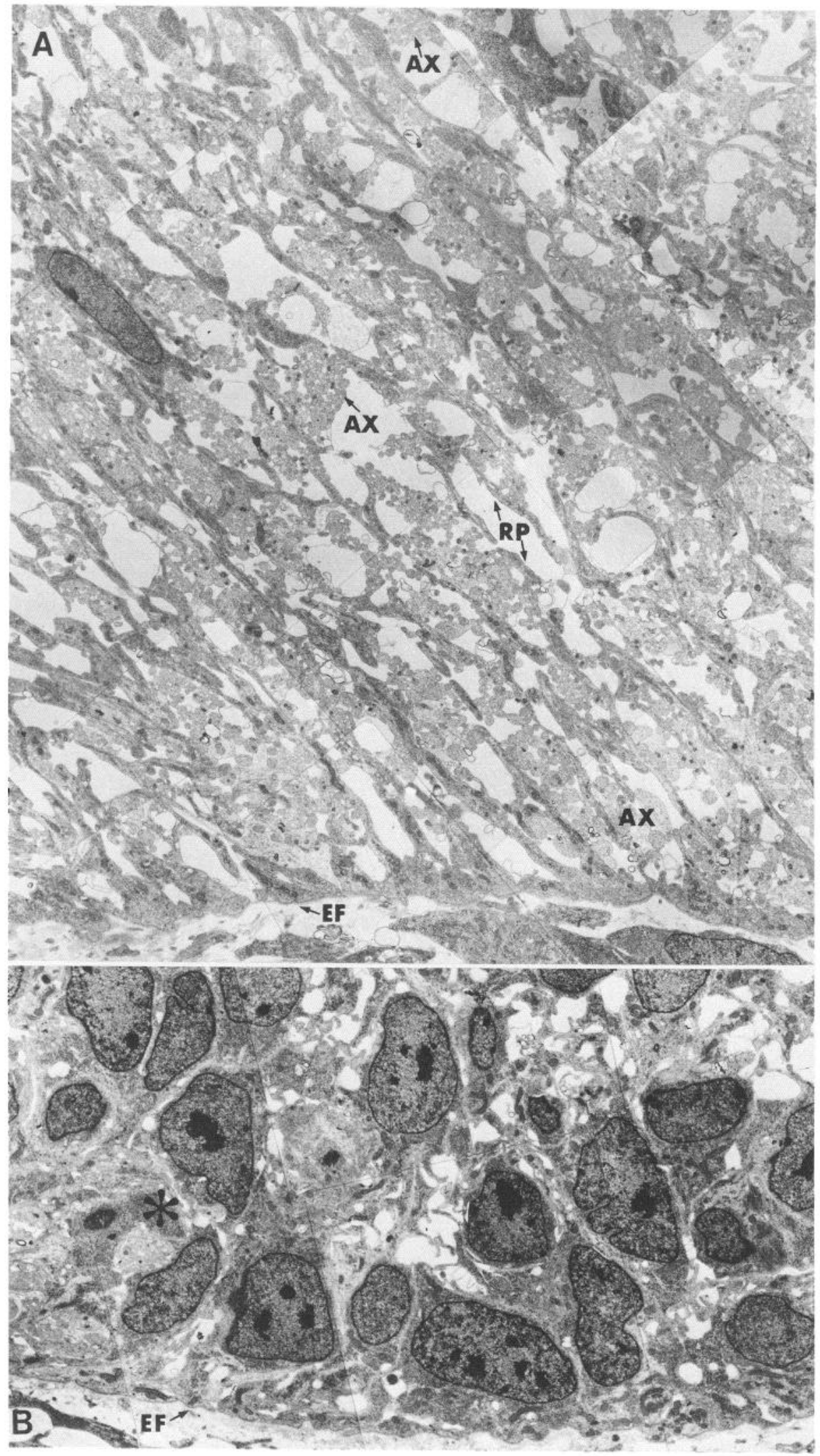

Figure 5. Electron micrographs of the optic tract region of the diencephalon $(A)$, compared with the knot region $(B)$ at the D/T junction of an E5 chick. Note the greater length and orientation of the radial processes of neuroepithelial support cells in the tract versus the disorganized nature of the cells in the knot. The rostralmost fascicle of axons at the chiasm is noted by an asterisk in $B$. In $B$, note, also, the lack of long radial processes and the presence of many cell bodies in the knot. $\times 7000 . A X$, Axons; $R P$, radial processes; $E F$, endfeet. the necrotic region at the pre-optic recess (the presumptive knot). The patchy distribution of N-CAM staining at the chiasm of day 2 embryos, although a pre-axonal stage for retinal ganglion cells, is nonetheless suggestive of the geometry of axon fascicles.
Support-cell staining in the chiasm becomes readily detectable during the following $2 \mathrm{~d}$, just at, or slightly before, the time when the optic fibers first arrive (Fig. 2,D,E). Thus, at E2, N-CAM staining at the base of the diencephalon caudal to the knot may 


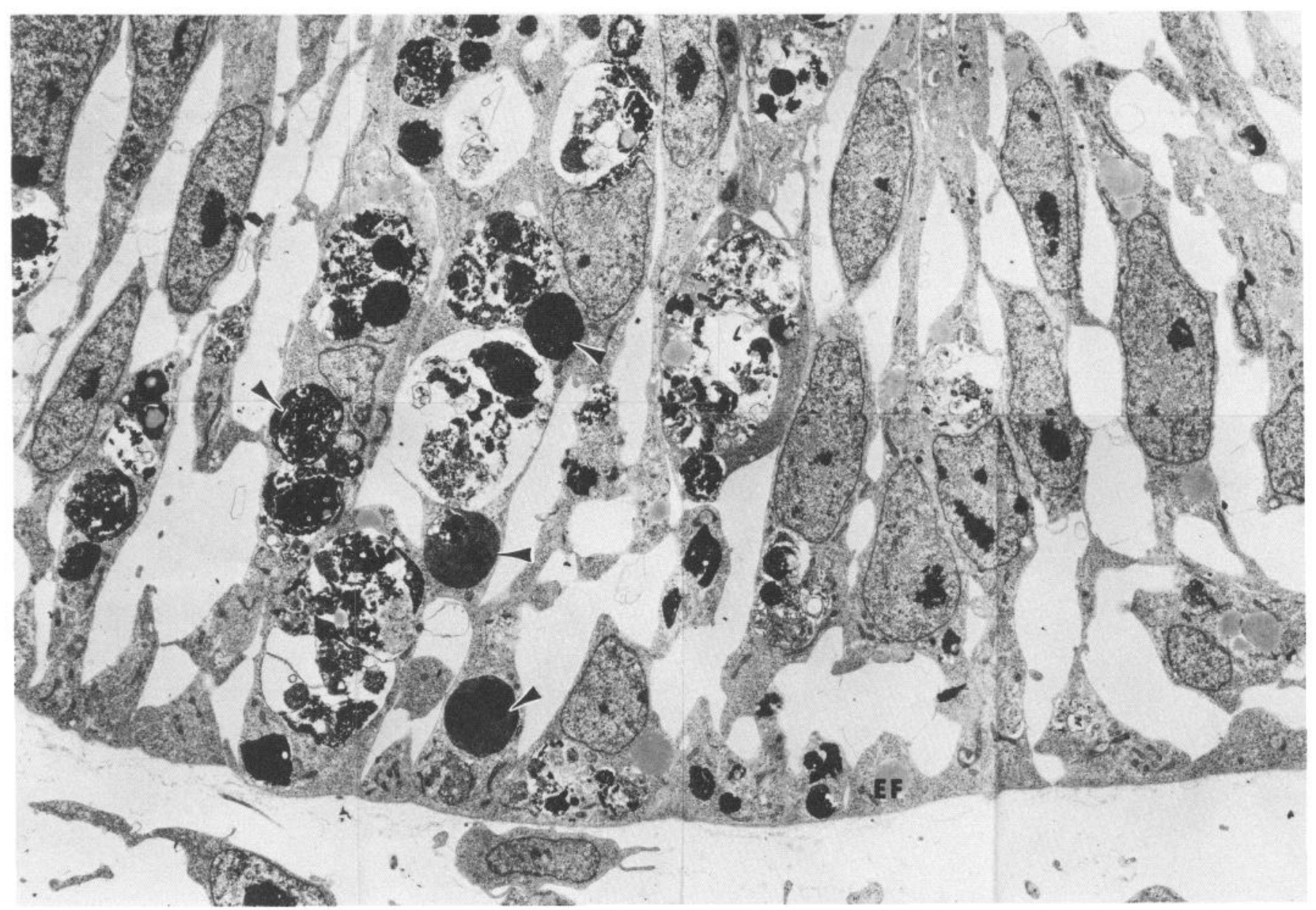

Figure 6. An electron micrograph of the $\mathrm{D} / \mathrm{T}$ junction (sagittal plane) of an embryonic day 2 chick (see Figs. $2, A, G$, for low magnification). Many dense pycnotic granules (arrowheads) are present in vacuoles within the cytoplasm of radial neuroepithelial cells that occupy the same position that will eventually become the knot and form the boundary between the centrally projecting axons of the olfactory and optic systems. $\times 12,000$. $E F$, Endfeet.

represent some other, non-optic axon system-most likely the supraoptic decussation - that uses the chiasm as part of its pathway, but that also respects the D/T border (Rogers et al., 1986).

On E3-4, additional N-CAM-stained patches appeared at the ventral margin of the chiasm. Unlike on E2, there were many axons located in the optic stalk at these later ages (not shown). The crescent-shaped distribution and marginal location of the staining at the chiasm are characteristic of optic axon fascicles adjacent to endfeet (Fig. 2,D,E). On embryonic days 3 and 4 , radial support-cell processes at the chiasm were also N-CAMpositive, particularly at the endfoot region (Fig. 2,D,E). However, the staining on neuroepithelial cells stopped abruptly at the border between the chiasm and the knot, where axons were lacking (Fig. 2D), and the knot remained N-CAM-free on embryonic day 5 as well (Fig. $3 D$ ). Within the tract, where we have observed long radial processes of support cells and spreading of axon fascicles, the staining for N-CAM extended far deeper into the tissue than at the chiasm.

Neuroepithelial support cells and axons along the route of the olfactory tract were also intensely N-CAM-positive on embryonic days 4 and 5 . On E4 the staining pattern was graded; it began most faintly and primarily marginally at the caudalmost end of the tract near the knot (i.e., near the growing front of the fibers), and increased in both intensity and radial spread in the rostral direction toward the olfactory bulb (Fig. $4, A, B$ ).

\section{Discussion}

A fundamental feature of the CNS is the highly stereotyped spatial arrangement of its long-projection axon pathways. Except for occasional aberrancies in location, each of the approximately 120 discrete projection tracts (estimated from De Armond et al., 1976), including the various lemnisci, funiculi, striata, etc., occupies a characteristic position within the brain and in relation to the others. In many regions, the long fiber tracts closely abut, intermix, or intersect. However, there are a number of boundary zones located in the CNS across which projection axons do not pass. When one charts the distribution of the axon-free territories, it becomes apparent that the least traveled route for long axons is across the midline, above the ventricular system. Therefore, with the obvious exception of the few confined regions containing the dorsal commissures, most of the tissue that stems from the vicinity of the original seam of the neural tube lacks projecting axon bundles. An important example of an axon tract boundary in this seam occurs at the rostroventral end of the lamina terminalis, at the dien- 
cephalic/telencephalic junction. Here, 2 major sensory axon pathways, the optic and olfactory, are segregated from each other (Kallen, 1954).

How is this axon boundary created during embryogenesis? Are there unique cellular and molecular strategies that have evolved to make this region actively repulsive or physically deflective to axons; or does this region simply lack the factors that are needed to promote growth within the pathway domains?

Our results suggest that at least 3 phenomena are peculiar to the discrete strip of neuroepithelium that will eventually form the D/T border: (1) the formation of a dense, nuclei-rich, "knotlike" structurc crcated by the diminution and disorientation of the marginal radial processes of neuroepithelial endfeet, as well as by the lack of marginal extracellular spaces; (2) the persistent absence (at least through E5) of N-CAM expression by this localized population of support cells; and (3) the initial presence of a massive cell death confined exactly to the region of this presumptive axonal boundary. In essence, the knot effectively separates 2 basal marginal zones where the central optic and olfactory axons will eventually emerge.

Do these changes at the $\mathrm{D} / \mathrm{T}$ boundary selectively alter the cellular and extracellular features of the marginal zone that have been postulated to be attractive to axons? It has been proposed that one particular part of the primitive pathway neuroepithelial cell-its pial endfoot and connecting radial process-become specialized during development, thus stimulating pioneering fibers to grow in discrete zones along the neural margin (Silver and Robb, 1979; Silver and Sapiro, 1981; Silver and Rutishauser, 1984). Thus, the great majority of the pioneering, as well as many, but not all, of the later-arriving optic fiber growth cones tend to migrate tangentially along the outer edge of the brain, moving from endfoot to endfoot as they grow toward their central targets (also see Rager and von Oeynhausen, 1979; Kuwada, 1986).

It has recently been suggested that the association between pioneering optic axons and endfeet may be mediated, in part, by N-CAM. Two observations have led to this proposal. First, the initial expression of N-CAM on neuroepithelial cells in certain sectors of the optic pathway is polarized at the endfoot surface and marginal radial processes, where most growth cones are found (Silver and Rutishauser, 1984). This result is consistent with the marginal positioning of early (i.e., leading) axons and N-CAM within the olfactory tract as well. In the chick, it remains to be established whether the distribution of N-CAM on certain "pathway" support cells is totally independent of the arrival of axons or represents a predisposition of specialized subpopulations (or subsectors) of neuroepithelial cells to express N-CAM when contacted at their endfeet by growth cones. However, in the embryonic frog spinal cord, it has recently been shown that enhanced expression of N-CAM on a discrete corridor of endfeet at the lateral margins occurs at pre-axonal stages (Balak et al., 1987). Moreover, injections of anti-N-CAM Fab into the early developing eye cup of chicks cause stereotyped neuroepithelial malformations and, in addition, cause optic fibers to dislocate from the glial limitans as they exit from the globe (Silver and Rutishauser, 1984). These observations illustrate the close developmental interaction that occurs between retinal ganglion cell axons and N-CAM-producing regions of the neuroepithelium. The case for this relationship is strengthened by our present observation that the knot lacks N-CAM. Not only is the D/T junction, where the knot resides, N-CAMfree prior to the arrival of axons of both olfactory and optic systems, but this sector of the neuroepithelium remains N-CAMnegative during later development, even as fibers pass nearby.

Several other unusual phenomena suggest that the reduction in levels of N-CAM is not the only alteration that occurs at the rostral chiasmatic boundary in advance of its encounter with axons. We have shown that an intense focal necrosis occurs precisely in the region of the $\mathrm{D} / \mathrm{T}$ junction. This particular necrotic locus, the so-called "suboptic death center," was first discovered by Ernst in 1926. In the years following its initial description, the suboptic center has received considerable attention because it is one of the most strictly localized and intense foci of degeneration in the early developing vertebrate brain. Ernst hypothesized that this particular zone might serve a morphogenetic function, being caused by and/or allowing for the ventral and forward shifting of the eye and optic stalk. However, Kallen (1965) showed that even after complete removal of the eye rudiment, the cell death continued to occur on schedule and with the same intensity. Kallen (1965) proposed, therefore, that the cells in this region werc genctically predestined to die. It is tempting to speculate that the cataclysmic cell death that occurs at the $\mathrm{D} / \mathrm{T}$ junction may not only serve to sculpt the pre-optic recess itself, but also trigger, or at least mark, the beginning of the cascade of events that leads to the formation of the dense, disorganized, and N-CAM-free knot of cells at the D/T border. Since the pycnotic debris that is generated there is phagocytosed locally by the remaining neuroepithelia, it is conceivable that the cell disruption and release of large amounts of lytic enzymes could provoke such an effect.

It is interesting that, during the degenerative phase and after, the density of tissue and number of cells within the $\mathrm{D} / \mathrm{T}$ junction become progressively greater, although one might have expected that removal of cells in an embryo would have an opposite effect. A similar, although transient, increase in cell density occurs as a wave of cell death sweeps through the early embryonic retina (Silver, 1976). However, in the suboptic center, unlike the retina, the cell death remains focused in one place. The significance of increased cell density and reduced extracellular space for creating an axon-refractory zone is further indicated by recent studies that suggest that extracellular spaces between neuroepithelial cells, where axons do grow, contain matrix substances that may promote or at least permit growth cone elongation (Derer and Nakanishi, 1983; Bignami and Delpech 1985; Bruckner et al., 1985; Hankin and Silver, 1986; Palmert et al., 1986). During later development, the knot continues to expand around, as well as into, the rostral surface of the chiasm (Silver, unpublished observations). Thus, in addition to forming an axonrefractory boundary, the knot may serve as a secondary germinal zone for the production of glial cells, most likely the progenitor cells of the oligodendrocyte-type 2 astrocyte lineage (Small, 1968) that invade the optic chiasm or nerve.

In summary, the knot lacks organized endfeet and associated extracellular spaces, as well as at least one adhesion molecule. It is also important to stress that N-CAM is not the only molecule that may control the positioning of optic axons within their pathway. Recent evidence, using anti-laminin antibodies on sections through the developing mouse optic system, suggests that laminin in the form of punctate deposits located preferentially near the endfeet of neuroepithelial cells may also be involved (P. Liesi and J. Silver, unpublished observations). We suggest that the modulation of any, or more likely of all of these parameters is involved in creating a barrier to growing axons at the floor of the diencephalic/telencephalic junction. 


\section{References}

Alvarado-Mallart, R. M., and C. Sotelo (1984) Homotopic and heterotopic transplantations of quail tectal primordia in chick embryos: Organization of the retinotectal projections in the chimeric embryos. Dev. Biol. 103: 378-398.

Balak, K., M. Jacobson, J. Sunshine, and U. Rutishauser (1987) Neural cell adhesion molecule expression in Xenopus embryos. Dev. Biol. 119: 540-550.

Bignami, A., and B. Delpech (1985) Extracellular matrix glycoprotein (Hyaluronectin) in early cerebral development: Immunofluorescence study of the rat cmbryo. Int. J. Dev. Ncurosci. 3: 301-307.

Brodal, A. (1981) Neurological Anatomy in Relation to Clinical Medicine, pp. 578-588, 640-652, Oxford U. P., New York.

Bruckner, G., L. Muller, L. Wollweber, R. Samtleben, and D. Biesold (1985) Lectin binding sites and anionic components related to differentiation in the prenatal rat cerebral cortex. J. Hirnforsch. 26:615634.

Constantine-Paton, M., and R. R. Capranica (1976) Axonal guidance of developing optic nerves in the frog. I. Anatomy of the projection from transplanted eye primordia. J. Comp. Neurol. 170: 17-32.

De Armond, S. J., M. M. Fusco, and M. M. Dewey (1976) A Photographic Atlas: Structure of the Human Brain, Oxford U. P., New York.

Derer, P., and S. Nakanishi (1983) Extracellular matrix distribution during neocortical wall ontogenesis in normal and "reeler" mice. $J$. Hirnforsch. 24: 209-224.

Ehrlich, D., and R. Mark (1984) The course of axons of retinal ganglion cells within the optic nerve and tract of the chick (Gallus gallus). J. Comp. Neurol. 223: 583-591.

Ernst, M. (1926) Uber untergang von zellen wahrend der normalen entwicklung bei wirbeltieren. Z. Ges. Anat. 79: 228-262.

Frelinger, III, A. L., and U. Rutishauser (1986) Topology of NCAM structural and functional determinants. II. Placement of monoclonal antibody epitopes. J. Cell Biol. 103: 1729-1737.

Frost, D. O. (1984) Axonal growth and target selection during development: Retinal projections to the ventrobasal complex and other "nonvisual" structures in neonatal Syrian hamsters. J. Comp. Neurol. 230: $576-592$.

Goldberg, S. (1974) Studies on the mechanics of the visual pathways in the chick cmbryo. Dev. Biol. 36: 24-43.

Hamburger, V., and H. L. Hamilton (1951) A series of normal stages in the development of the chick embryo. J. Morphol. 88: 49-82.

Hankin, M. H., and J. Silver (1986) Mechanisms of axonal guidance: The problem of intersecting fiber systems. In Developmental Biology: A Comprehensive Synthesis, Vol. 2: The Cellular Basis of Morphogenesis, L. Browder, ed., pp. 565-599, Plenum, New York.

Harris, W. A. (1980) Regions of the brain influencing the projection of developing optic tracts in the salamander. J. Comp. Neurol. 194 319-333.

Harvey, A. R., and R. D. Lund (1981) Transplantation of tectal tissue in rats. II. Distribution of host neurons which project to transplants. J. Comp. Neurol. 202: 505-520.

Herrick, C. J. (1941) Development of the optic nerves of Amblystoma. J. Comp. Neurol. 74: 473-529.

Holt, C. E. (1984) Does timing of axon outgrowth influence initial retinotectal topography in Xenopus? J. Neurosci. 4: 1130-1152.

Kallen, B. (1954) The embryology of the telencephalic fibre systems in the mouse. J. Embryol. Exp. Morphol. 2: 87-100.

Kallen, B. (1965) Degeneration and regeneration in the vertebrate CNS during embryogenesis. Prog. Brain Res. 14: 77-96.

Katz, M. J., and R. J. Lasek (1979) Substrate pathways which guide growing axons in Xenopus embryos. J. Comp. Neurol. 183: 817-832.
Krayanek, S. (1980) Structure and orientation of extracellular matrix in developing chick optic tectum. Anat. Rec. 197: 95-109.

Kuwada, J. (1986) Cell recognition by neuronal growth cones in a simple vertebrate embryo. Science 233: 740-746.

Lund, R. D., and A. H. Bunt (1976) Prenatal development of central optic pathways in albino rats. J. Comp. Neurol. 165: 247-264.

Maggs, A., and J. Scholes (1986) Glial domains and nerve fiber patterns in the fish retinotectal pathways. J. Neurosci. 6: 424-438.

McLoon, S. C., and R. D. Lund (1982) Transient retinofugal pathways in the developing chick. Exp. Brain Res. 45: 277-284.

O'Leary, D. D. M., C. R. Gerfen, and W. M. Cowan (1983) The development and restriction of the ipsilateral retinofugal projection in the chick. Dev. Brain Res. 10:93-109.

Palmert, M. R., M. J. Kujawa, A. I. Caplan, and J. Silver (1986) Role of extracellular matrix and endfoot "tangles" in axonal patterning of the avian telencephalon. Soc. Neurosci. Abstr. 12: 1334

Pickard, G. E., and A.-J. Silverman (1981) Direct retinal projections to the hypothalamus, piriform cortex, and accessory optic nuclei in the golden hamster as demonstrated by a sensitive anterograde HRP technique. J. Comp. Neurol. 196: 155-172.

Rager, G., and B. von Oeynhausen (1979) Ingrowth and ramification of retinal fibers in the developing optic tectum of the chick embryo. Exp. Brain Res. 35: 213-227.

Rogers, L. J., T. Robinson, and D. Ehrlich (1986) Role of the supraoptic decussation in the development of asymmetry of brain function in the chicken. Dev. Brain Res. 28: 33-39.

Schwenk, G. C., and E. Hibbard (1977) An autoradiographic study of optic fiber projections from eye grafts in eyeless mutant axolotls. Exp. Neurol. 55: 498-507.

Silver, J. (1976) A study of ocular morphogenesis in the rat using $\left[{ }^{3} \mathrm{H}\right]$ thymidine autoradiography. Evidence for thymidine recycling in the developing retina. Dev. Biol. 49: 487-495.

Silver, J. (1984) Studies on the factors that govern directionality of axonal growth in the embryonic optic nerve and at the chiasm of mice. J. Comp. Neurol. 223: 238-251.

Silver, J., and R. M. Robb (1979) Studies on the development of the eye cup and optic nerve in normal mice and in mutants with congenital optic nerve aplasia. Dev. Biol. 68: 175-190.

Silver, J., and U. Rutishauser (1984) Guidance of optic axons in vivo by a preformed adhesive pathway on neuroepithelial endfeet. Dev. Biol. 106: 485-499.

Silver, J., and J. Sapiro (1981) Axonal guidance during development of the optic nerve: The role of pigmented epithelia and other extrinsic factors. J. Comp. Neurol. 202: 521-538.

Silver, J., G. M. Smith, R. H. Miller, and P. R. Levitt (1985) The immature astrocyte: Its role during normal CNS axon tract development and its ability to reduce scar formation and promote axonal regeneration when transplanted into the brains of adults. Neurosci. Abstr. 11: 334.

Small, R. K. (1986) Evidence that progenitor cells of the oligodendrocyte type-2 astrocyte lineage migrate into the developing rat optic nerve. Soc. Neurosci. Abstr. 12: 183.

Steedman, J. G., R. U. Stirling, and R. M. Gaze (1979) The central pathways of optic fibers in Xenopus tadpole. J. Embryol. Exp. Morphol. 50: 199-215.

Stone, L. S. (1960) Polarization of the retina and development of vision. J. Exp. Zool. 145: 85-93.

Watanabe, M., A. L. Frelinger, III, and U. Rutishauser (1986) Topology of NCAM structural and functional determinants. I. Classification of monoclonal antibody epitopes. J. Cell Biol. 103: 17211727. 